\title{
Mucinous Cystadenocarcinoma in Breast: Complicated with Lymph Node, Thoracic Wall Metastasis, and Contralateral Triple Negative Breast Cancer: A Case Report
}

\author{
Zhenyu Li \\ Chongqing University \\ Qingming Jiang \\ Chongqing University \\ Dongfang Guo \\ Chongqing University \\ Yong Cao \\ Chongqing University \\ Yangling Peng \\ Chongqing University \\ Jing Zhang \\ Chongqing University \\ Juanhui Dong ( $\square$ dongjuanhui1984@163.com ) \\ Chongqing University
}

\section{Case Report}

Keywords: Mucinous cystadenocarcinoma, metastasis, prognosis, triple negative breast cancer, case report

Posted Date: September 27th, 2021

DOI: https://doi.org/10.21203/rs.3.rs-898549/v1

License: (c) (1) This work is licensed under a Creative Commons Attribution 4.0 International License. Read Full License 


\section{Abstract}

Background: Mucinous cystadenocarcinoma of the breast is a very rare and special type adenocarcinoma of the mammary tract. We report a rare case of mucinous cystadenocarcinoma of the breast with an unfavourable prognosis which was confirmed after surgical resection pathologically.

Case summary: At low power appearance, the tumor formed mucus-filled spaces of varying sizes, and with mucus-rich tumor cells lining the walls. The tumor cells were arranged in papillary structures. At high magnification, the tumor cells shown moderate to severe atypia, which were most of simple columnar cells with nuclei in the base and cytoplasm rich in mucin. In some areas, tumor cells proliferated, stratified, and clustered that protruded into the cyst cavity, or formed papillary structures with thin fibrovascular core. Immunohistochemical staining showed cytoplasm CK7 strong positive of tumor cells and cytoplasm negative of CK20, PAX-8 and CDX2, which could exclude metastatic tumors from ovary and intestine. And the tumor cells also demonstrated the basal-like characters such as negative for ER, PR, HER-2 (triple-negative), CK5/6 focal positive, EGFR positive. Besides, a triple negative breast cancer with basal-like features, lymph node, thoracic wall metastasis of mucinous cystadenocarcinoma was found.

Conclusion: Mucinous cystadenocarcinoma of the breast usually have a favorable prognosis, but in this case, it happened lymph node, thoracic wall metastasis, therefore, it needs extra attention of clinical workers.

\section{Introduction}

Mucinous cystadenocarcinoma (MCA) is a very rare breast tumor. This type of tumor mainly occurs in the ovaries $^{[1]}$. To our knowledge, almost only 32 relevant literature have been published in National Center for Biotechnology Information (NCBI) database so far, mostly focusing on the pathologic and radiographic findings since first authoritatively introduced at $1998^{[2,3,4,5]}$. MCAs are considered to have a distinct pathogenesis and biologic behavior, they should be distinguished from ordinary mucinous carcinomas, cystic hypersecretory carcinomas, and carcinomas of other histologic subtypes ${ }^{[6,7]}$. The lymph node and distant metastasis was very rare in MCA of the breast. Here, we present a case report of MCA in breast, complicated with lymph node, thoracic wall metastasis, and contralateral triple negative breast cancer.

\section{Case Presentation}

A 53 years old female was found to have double breast neoplasm in local hospital during physical examination more than 1 years ago. The invasive breast cancer in both sides of the diagnosis was made at the time. The patient was received a neoadjuvant chemotherapy $(120 \mathrm{mg}$ docetaxel, $800 \mathrm{mg}$ cyclophosphamide and $800 \mathrm{mg}$ doxorubicin) for four cycles. Regrettably, the tumor continued to grow, and then the patient transfered to our hospital for further treatment. The patient had no familial genetic, 
psychosocial and exposure to toxins history. Furthermore, he was conducted in line with the care checklist.

Laboratory tests of the tumor biomarkers such as CA125 (34.70 U/ml), CA153 (23.40 U/ml), CA724 (6.98 $\mathrm{mlU} / \mathrm{ml})$ and CEA $(71.20 \mathrm{ng} / \mathrm{ml})$ showed slightly higher.

Then, the patient was admitted and underwent chest computed tomography (CT) and magnetic resonance imaging (MRI). The $\mathrm{CT}$ demonstrated bilateral breast masses, multiple enlarged lymph nodes and increased metabolism of the masses (Fig. 1A). The MRI shown that a large mass in the outer part of the right breast, and invaded the chest wall and skin. New skin nodules were detected in the inner part of the right breast, nodules in the axillary and caudal region of the right breast, and a mass of the left breast (Fig. 1B-C). In addition, pelvic MRI showed no lesions (Fig. 1D).

To verify the previous diagnosis from the local hospital, puncture biopsy of the breast mass of both sides was performed. An invasive carcinoma of left breast and an invasive carcinoma with mucus-rich of right breast was detected (Fig. 2A-B).

The patient was received a new neoadjuvant chemotherapy (40mg d1, d8, navelbine, $50 \mathrm{mg} \mathrm{d1}$,lobaplatin) for two cycles. The tumor was significantly shrink and became soft, and then, the radical mastectomy of both breasts was performed.

The mass about $4 \times 3.5 \times 2.5 \mathrm{~cm}^{3}$ in size of the left breast. The histological characteristics were hard and with unclear boundaries. At lower magnification, tumor cells were arranged in cords, clusters, some areas the growth pattern was map-like pattern with central necrosis. At high magnification, the tumor cells were large, the nucleoli were easily visible (about $22 / 2 \mathrm{~mm}^{2}$ ). The histopathological type was invasive breast carcinoma of no special type, Nottingham grade 3. There was little stroma, no clear inflammatory cell infiltration, and no clear withdrawal response in the tumor. Reaction grading after neochemotherapy (Miller-payne system): G1. Regional lymph nodes dissection showed no definite metastasis $(0 / 36)$. Immunohistochemical staining showed that the tumor cells were negative for ER, PR and HER2 (Fig. 3CE)

In particularly, the macroscopic observation showed that the huge mass almost occupied the whole right breast, about $15 \times 13.5 \times 10 \mathrm{~cm}^{3}$, with a little of normal compressed breast tissue remaining around. Multiple ulcerations were seen on the surface of the skin, accompanied by a large amount of necrosis and purulent secretions. The section of the mass was cystic and solid, filled with translucent to bloody mucus, and the solid area was pale and gelatinous.

Histopathological findings of the right resected mass revealed the tumor formed mucus-filled spaces of varying sizes, and with mucus-rich tumor cells lining the walls. The tumor cells were arranged in papillary structures of low power appearance. At high magnification, the tumor cells showed moderate to severe atypia, which were most of simple columnar cells with nuclei in the base and cytoplasm rich in mucin. In some areas, tumor cells proliferated, stratified, and clustered that protruded into the cyst cavity, or formed 
papillary structures with thin fibrovascular core. Ductal carcinoma in situ was seen around the peripher of the tumor (Fig. 4A-B). Response grading after neoadjuvant chemotherapy (Miller-payne system) was Grade 1. Immunohistochemical staining showed that the tumor cells were negative for ER, PR and HER2 (Fig. 4C-E). MCA metastasis was observed in the right axillary lymph node (8/26, Fig. 4F).

Immunohistochemical staining showed that the tumor cells were positive for CK5/6 (focal), CK7 and EGFR (Fig. 5A-C), but negative for CK20, CDX2, and PAX8 (Fig. 5D-F).

In addition, thoracic wall metastasis of right MCA was detected (Fig. 6A). Immunohistochemical staining showed that the tumor cells were negative for ER, PR, HER2 and GATA-3 (Fig. 6B-E).

The process of the thoracic wall metastasis from mammary MCA was conjectured in this case, the migration and invasion of the cancer cells break through basement membrane and went through endothelial cells of related vessels, and then transfered into lymph-vessel. Hence, further metastases to the thoracic wall and skin (Fig. 7). This process required further studies by clinicians.

The final diagnosis was made by Dr. Juanhui Dong, Dr. Zhenyu Li, Dr. Qingming Jiang, and Dr. Dongfang Guo of the department of pathology in Chongqing University Cancer Hospital.

The patient remained under careful observation by endoscopy and CT follow-up, and there was no recurrence or metastasis in the 6 months follow-up. The patient got appropriate perspective including the assessment and the episode of care in every 3 months.

\section{Discussion}

MCA of the breast has a favorable prognosis cancer of invasive breast carcinoma with more than 40 cases reported in the literatures ${ }^{[8,9,10]}$. Microscopically, the tumors were characterized by cystic spaces lined by predominantly bland-appearing columnar mucinous cells with stratification, tufting, and papillary formations. and immunohistochemical staining failed to show immunoreactivity for ER and PR. furthermore shows positively with CK7 but negatively with CK20 $[2,11]$. In our case, the morphology of the tumor cells and growth pattern was consistent with previous reports ${ }^{[2,5,9]}$. The right resected mass revealed the tumor formed mucus-filled spaces of varying sizes, and with mucus-rich tumor cells lining the walls. The tumor cells were arranged in papillary structures and showed moderate to severe atypia. Immunohistochemical staining showed that the tumor cells were negative for ER, PR and HER2. MCA metastasis was observed in the right axillary lymph node. Contrary to other reports, we found 8 right axillary lymph nodes metastasis in this case which may indicate a poor prognosis. To verify whether the tumor was coming from the ovary and intestine, MRI and immunohistochemical staining for CDX2 and PAX8 was involved and shown the negative outcomes.

Interestingly, the contralateral side of MCA existed a triple negative breast cancer, we can not recognize which came first. Furthermore, the triple negative breast cancer which is considered to have a bad 
prognosis of the left side had no lymph node metastasis in our case, whereas the MCA which is considered to have a favorable prognosis ${ }^{[6,12,13]}$.

It has no reported that MCA can appear thoracic wall metastasis yet. Our case will be the first to report that MCA also can have a poor prognosis. As for the cause of thoracic wall metastasis, we conjectured, migration and invasion of the cancer cells break through basement membrane and into endothelial cells of related vessels, and transfered into lymph-vessel. And then, further metastases to the thoracic wall and skin. This process required further studies by clinicians.

In summary, we present an unusual and rare case of MCA complicated with lymph node, thoracic wall metastasis, and contralateral triple negative breast cancer which is first reported. But we did not use the genetic detection to detect the gene mutation, that was the limitation of this case. Although the tumor has distinct histological and immunohistochemical features, awareness is important for its diagnosis and prognosis. It is worth mentioning that MCA also can have a poor prognosis. Finally, the patient was satisfied with the treatment plan, process and prognosis, and will continue to follow up as prescribed by the doctors.

\section{Abbreviations}

MCA

Mucinous cystadenocarcinoma; CT:computed tomography; MRI:magnetic resonance imaging; CK:cytokeratin; ER:estrogen receptor; PR:progesterone receptor; HER2; human epidermal growth factor receptor 2; PAX-8:paired box 8; CDX2:caudal type homeobox 2; EGFR:epidermal growth factor receptor; GATA-3:GATA binding protein 3; CEA:carcinoembryonic antigen; NCBI:National Center for Biotechnology Information.

\section{Declarations}

\section{Ethics approval and consent to participate}

Informed consent was obtained in this case, and protocols were approved by the Ethics approval of Chongqing University Cancer Hospital. The patient provided informed consent for the publication of this report and any accompanying images.

\section{Availability of data and materials}

The datasets used or analysed during the current study are available from the corresponding author on reasonable request.

\section{Competing interests}

The authors declare that there is no conflict of interest. 


\section{Funding}

No funding information.

\section{Author's contributions}

ZL contributed to acquisition, analysis and interpretation of patient data and the drafting of the manuscript. YC contributed to the treatment and follow-up of the patient. YP and DG contributed to the acquire the CT and MRI data. JZ contributed to the immunohistochemistry methods. QJ and JD gave the final approval of the report. All authors read and approved the final manuscript.

\section{Acknowledgements}

Not applicable.

\section{References}

1. Taylor EC, Irshaid L, Mathur M. Multimodality Imaging Approach to Ovarian Neoplasms with Pathologic Correlation. Radiographics. 2021;41(1):289-315.

2. Koenig C, Tavassoli FA. Mucinous cystadenocarcinoma of the breast. Am J Surg Pathol. 1998;22(6):698-703.

3. Domoto H, Terahata S, Yamazaki T, et al. Mucinous cystadenocarcinoma of the breast showing sulfomucin production. Histopathology. 2000;36(6):567-9.

4. Hu Y, Tian C, Zhang X, et al. 18F-FDG PET/CT Findings in a Patient With Primary Mucinous Cystadenocarcinoma of the Breast. Clin Nucl Med. 2020;45(2):159-60.

5. Jain E, Kumar A, Jain R, et al. Primary Mucinous Cystadenocarcinoma of the Breast: A Rare Case Report With Review of Literature. Int J Surg Pathol, 2021, 1066896921991650.

6. Honma N, Sakamoto G, Ikenaga M, et al. Mucinous cystadenocarcinoma of the breast: a case report and review of the literature. Arch Pathol Lab Med. 2003;127(8):1031-3.

7. Chen WY, Chen CS, Chen HC, et al. Mucinous cystadenocarcinoma of the breast coexisting with infiltrating ductal carcinoma. Pathol Int. 2004;54(10):781-6.

8. Coyne JD, Irion L. Mammary mucinous cystadenocarcinoma. Histopathology. 2006;49(6):659-60.

9. Wang X, Li Y, Zhao P, et al. Primary mucinous cystadenocarcinoma of the breast: a clinicopathologic analysis of one case and review of the literature. Int J Clin Exp Pathol. 2020;13(10):2562-8.

10. Sun M, Su S, Liu Q, et al. Mammary synchronous mucinous cystadenocarcinoma and columnar cell mucinous carcinoma: a case report. Int J Clin Exp Pathol. 2020;13(9):2381-6.

11. Deng $Y$, Xue D, Wang $X$, et al. Mucinous cystadenocarcinoma of the breast with a basal-like immunophenotype. Pathol Int. 2012;62(6):429-32.

12. Chen $\mathrm{H}$, Wang T, Li K, et al. Effects of surface modification of quantum dots on viability and migration of triple-negative breast cancer cells. J Colloid Interface Sci. 2017;485:51-8. 
13. Huo D, Ikpatt F, Khramtsov A, et al. Population differences in breast cancer: survey in indigenous African women reveals over-representation of triple-negative breast cancer. J Clin Oncol. 2009;27(27):4515-21.

\section{Figures}

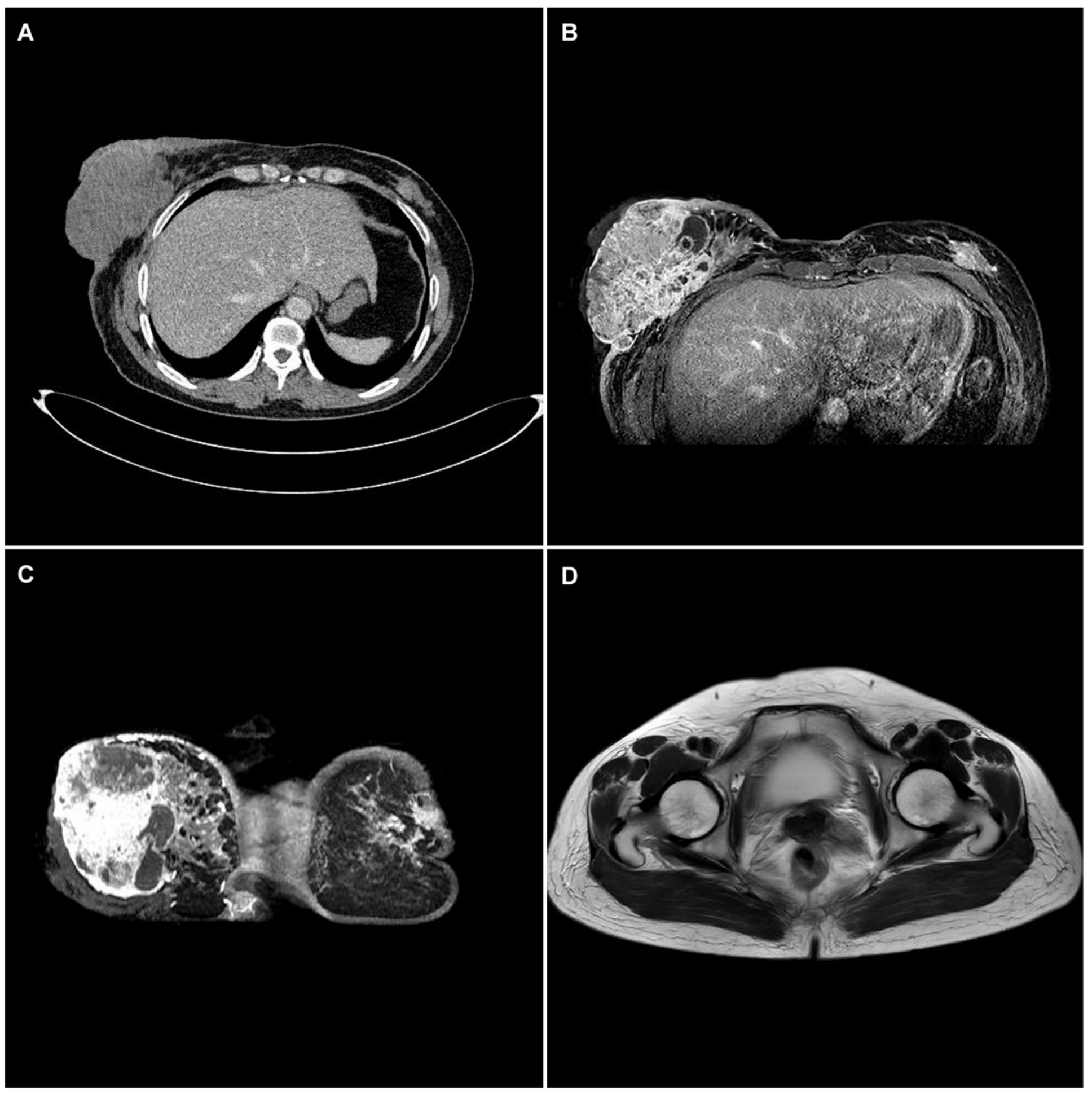

Figure 1 
(A) The lesion exhibited with bilateral breast masses and increased metabolism in axial unenhanced CT image. (B-C) A large mass in the outer part of the right breast, and invaded the chest wall and skin, and a mass of the left breast in MRI. (D) The pelvic MRI of the patient.

\section{Figure 2}

(A) The puncture biopsy of the breast mass of left breast $(\times 40)$, and (B) mucus-rich breast mass of right breast $(\times 40)$.

展

\section{Figure 3}

(A) The invasive breast carcinoma of left breast $(\times 10)$. (B) The arranged, growth pattern and nucleoli of the the invasive breast carcinoma ( $\times 200)$. Immunohistochemical staining $(\times 200)$ was negative for ER (C), PR (D), and HER2 (E).

\section{Figure 4}

(A) The mammary MCA of right breast $(\times 10)$. The mucus-rich tumor cells, structures, and the cyst cavity of the mammary MCA (×200). (B) Immunohistochemical staining ( $\times 200)$ was negative for ER (C), PR (D), and HER2 (E). MCA metastasis in the right axillary lymph node $(\times 10)$.

\section{Figure 5}

Immunohistochemical staining ( $\times 200)$ shown the tumor cells were positive for CK5/6 (A), CK7 (B) and EGFR (C), and negative for CK20 (D), CDX2 (E) and PAX-8 (F).

展

\section{Figure 6}

(A) Thoracic wall metastasis of right mammary MCA $(\times 10)$. Immunohistochemical staining $(\times 200)$ was negative for ER (B), PR (C), HER2 (D) and GATA-3 (E). 


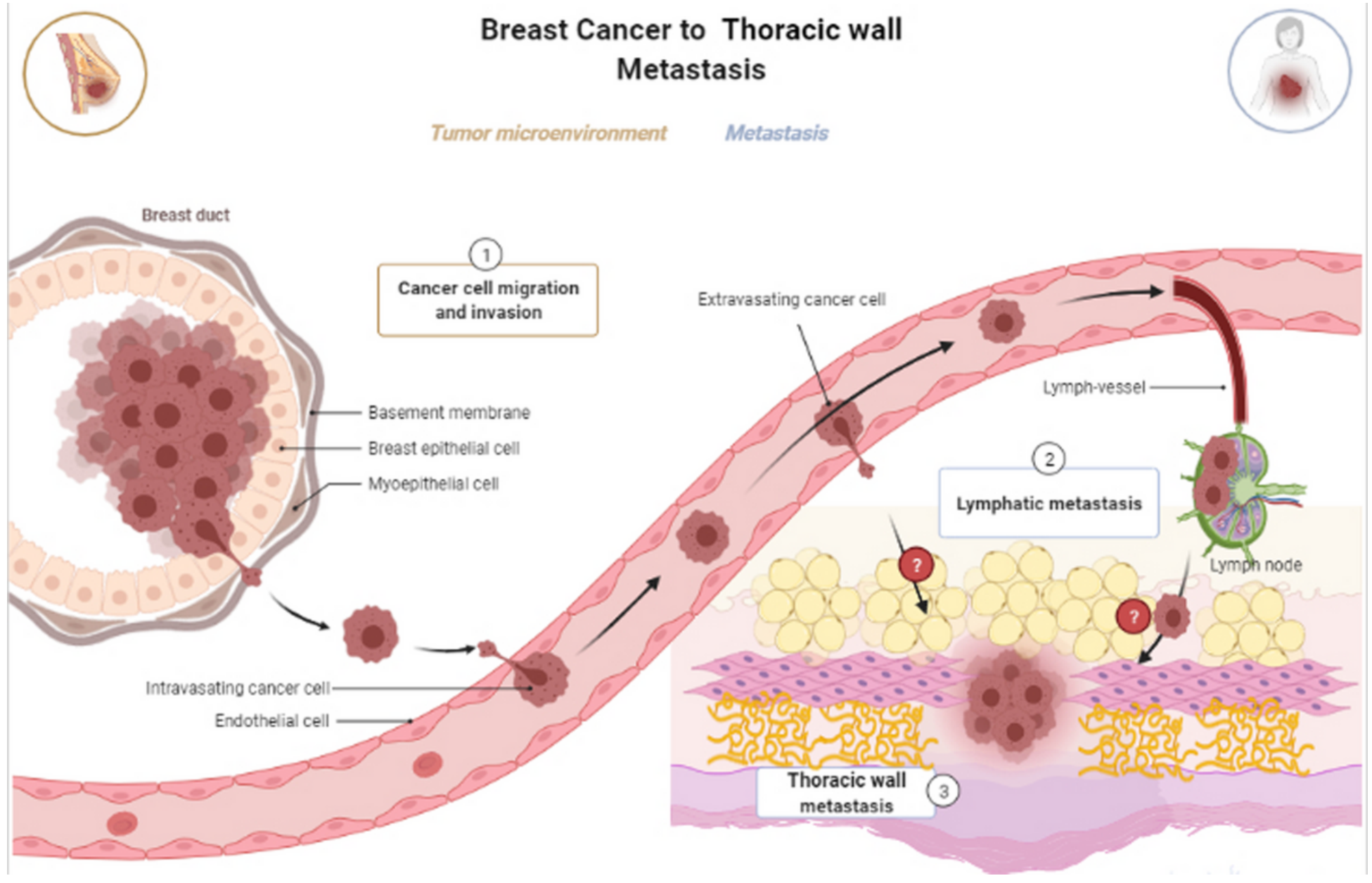

\section{Figure 7}

The conjectured process of thoracic wall metastasis of mammary MCA in this case.

\section{Supplementary Files}

This is a list of supplementary files associated with this preprint. Click to download.

- CAREchecklistEnglish2013.pdf 